\title{
Streamlining Management Information Systems Processes towards Improving Sustainability Reporting for a Tourism Company
}

\author{
By Martie Mearns ${ }^{*}$
}

\begin{abstract}
Information systems are used to allow organisations to achieve business objectives and are critical in supporting organisations in operational excellence, to develop new products or services, to improve decision-making or to gain a competitive advantage. Reporting on information is inevitably part of organisation's objectives regardless of the industry in which the organisation resides. Management information systems (MIS) are used to support decision-making in organisations. The most important characteristics of MIS are relevance, accuracy, usefulness, timeliness and completeness. When MISs adheres to these characteristics, decision makers in the organisation have confidence in their decisions resulting in desired actions. This paper reports on sustainability information reporting challenges of a luxury experiential travel company operating in geographically dispersed locations. The challenges are due to apparent disparate data captured on different systems used for financial, operational and sustainability reporting. Besides the disparate data generated, the different systems duplicate the same information on various versions of data sets on different platforms. Bandwidth capacity and varying business priorities complicates compliance of operational units' reporting. The research objective was to determine how current reporting into the MIS can be streamlined to ensure improved flow of relevant, accurate, useful, timely and complete sustainability data to facilitate optimal decision-making capabilities for various operational units. The case organisation's MIS practices were compared against a publicly trading peer organisation to benchmark the trends in sustainability reporting. Through a number of key interviews, the research results showed the information flows, flaws and gaps in the current reporting practices. The inherent differences in the case organisation and the peer organisation highlighted potential areas for improvement and change. The technology, organisation, environment (TOE) framework proved an ideal fit as a foundation for understanding the requirements to streamline the reporting process of MIS processes for the case organization.
\end{abstract}

Keywords: Management Information Systems, Technology Organisation Environment Framework, TOE Framework, sustainability information reporting, travel companies.

\section{Introduction}

The late Nelson Mandela said "ultimately conservation is about people. If you don't have sustainable development around wildlife parks - then the people will have no interest in them and the parks will not survive." Since the 1980s sustainable development with the environmental, social and economic pillars, has been at the forefront in organisations' operational endeavours. Much development has taken place and today organisations are mandated, accountable and

*Senior Research Associate, Universityof Johannesburg, South Africa. 
responsible to not only report on their sustainability matters, but also to put strategies in place that will continuously improve their sustainability.

Publicly listed companies, such as those trading on the Johannesburg Stock Exchange (JSE) in South Africa and elsewhere in the world are mandated to report on their sustainability matters according to prescribed reporting standards or indices. The Global Reporting Initiative (GRI) (2018) has developed sustainability reporting standards that are used by $93 \%$ of the world's largest 250 corporations. Hunter and Mearns (2014) indicate the GRI as the highest reporting standard in the world, being the most credible guidelines globally and considered a key resource of guidance for sustainability reporting. In 2004 South African publicly trading organisations used the Social Responsible Investment Index (SRI Index) which has since been replaced by the Environment, Social and Governance (ESG) disclosure indicators and data collection methodology of FTSE Russell (JSE 2015). The ESG provides organisations, investors and stakeholders with data analytics, ratings and indices important to investment and risk management decisions. These report findings provide the evidence needed to assist in managing exposure to environmental, social and governance aspects, to meet mandated stewardship requirements, to integrate ESG data into securities and portfolio analysis and to implement ESG-aware investment strategies (FTSE Russel 2016: 1). Even when companies are not publicly listed, reporting on ESG matters reflect a responsible approach to organisations' relationship with the environment and affected communities in their endeavours to make a profit.

The case organization that this paper reports on is not a listed company and operates in the tourism industry, managing operations mostly in southern and eastern Africa. In order for this organisation to meet the standards and requirements of responsible tourism, the South African National Standard (SANS) 1162:2016 (SANS 2016) may also be applicable to the manner in which they manage their ESG aspects. According to SANS 1162:2016 responsible tourism requirements are:

a. a proactive approach by tourism industry partners to develop, market and manage the tourism industry in a responsible manner, so as to create a competitive advantage;

b. the responsibility of the tourism industry to the environment through the promotion of balanced and sustainable tourism as it focuses on the development of environmentally-based tourism activities (such as game viewing and diving);

c. the responsibility of government and business to involve the local communities that are near thetourism plant and attractions through the development of meaningful economic linkages (forexample the supply of agricultural produce to the lodges, and outsourcing of laundry);

d. the responsibility to respect, invest in and develop local cultures and protect them from over commercializationand overexploitation;

e. the responsibility of local communities to become actively involved in the tourism industry, topractice sustainable development and to ensure the safety and security of the visitors; 
f. the responsibility to visitors through ensuring their safety, security and health;

g. the responsibility of both employers and employees in the tourism industry to each other as well as to the customer. Responsible trade union practices and responsible employment practices willbe the hallmarks of the new tourism in South Africa;

h. the responsibility of government as well as the tourists themselves to observe the norms and practices of South Africa, particularly with respect to the environment and culture of the country.

Tourism organisations that are therefore committed to environmental integrity, social justice and economic prosperity may therefore use responsible tourism standards as a pathway to achieve the goals of sustainable development. In any of these reporting initiatives whether organisations are using the GRI, the FTSE Russell ESG or the SANS responsible tourism standards asa guideline, data and information on the organisation's environmental, social and economic aspects has to be collected for reporting purposes.

The case organization, which will remain anonymous in this paper and will be referred to as The Case, claimed that disparate data was being generated for environmental reporting that differed from their financial data. Besides the disparate data being reported, different systems were used for financial and operational reporting. This resulted in potential increased incidences of data capture errors as well as duplication of effort. Research was therefore conducted at The Case organisation to determine how reporting into the management information systems (MIS) can be streamlined, to ensure better flow of data that can facilitate optimal decision-making capabilities for various operational and managerial units. Furthermore, a peer organisation (referred to as The Peer) was investigated to benchmark reporting capabilities. In order to conduct the investigation, the following research question was addressed:

How can the current state of The Case's Management Information System (MIS) be streamlined to facilitate optimal decision-making capabilities within the organisation?

The stated research objectives to operationalize the research question, were to determine the current information flows and reporting processes into the various centralised systems from certain remote locations; to investigate the best practices for information flows and reporting into a MIS for The Peer organisation and to establish requirements and quick wins to streamline the reporting process of management information for The Case.

\section{Literature Review}

The literature reviewed for this paper included a discussion on management information systems and the technology organisation environment (TOE) framework. 


\section{Management Information Systems}

When the Journal of Management Information Systems was launched in 1984 Zwass (1984) conducted a study on management information systems (MIS) as a practical and scholarly discipline and reported on developmental trends and focal points of MIS. MIS is devoted to the investigation and development of information systems in support of managerial functions (Zwass 1984:4) specifically to support the decision-making process. The agents involved in MIS are the users, developers, the organisation as well as the relationship with the information system itself.

Information systems and technologies are transforming the global business environment (Laudon and Laudon 2018: 33). Global firms and governments spent $€ 3.4$ trillion on information systems hardware, software and telecommunications equipment in 2015. Even considering this kind of spending, Laudon and Laudon (2018: 43) still claim that many business managers operate in an "information fog bank, never really having the right information at the right time to make an informed decision".Effective information systems should enable managers to rely on information to use real-time and data trends to make decisions. While an information system is defined as a set if interrelated components that collect or retrieve, process, store and distribute information to support decision-making and control in an organisation, an MIS deals with the behavioral issues as well as technical issues surrounding the development, use and impact of information systems used by managers and employees in the firm (Laudon and Laudon 2018: 44, 46).An organisation can therefore have a very powerful information system installed, however, might not be able to retrieve any information from it due to ineffective user behaviour regarding data capture or distribution. In order to use information systems effectively it is thus necessary to understand the organisation, management, and technology that shapes the system (Laudon and Laudon 2018: 47). The issues that are therefore prevalent to consider when discussing the effectiveness of MIS, are aspects such as technology acceptance of users, organisational structure and performance, trust issues, knowledge management, measurement issues, innovation and success factors (Shiau et al. 2015: 157). These issues include not only technical matters, but also behavioral approaches in terms of the users and their processes involved in capturing, adding value to and disseminating data and information via information systems. Changes in attitude, management, organisational policy and behaviour, and not only technological aspects (Laudon and Laudon 2018: 57) are often necessary for an information system to be an effective decision-making tool. For this reason, the Technology Organisation Environment (TOE) Framework proved an ideal fit as a foundation for understanding the requirements to streamline the reporting process of MIS processes for The Case organisation. 


\section{Technology Organisation Environment (TOE) Framework}

During the process of innovation, including the adoption and implementation stages of the innovation process, there are three elements that influence an organisation's adoption decisions. These elements are the technological, organisational and environmental contexts (Baker 2011).

From the technological context the organisation's existing technologies will set a limit on the scope and pace of technological change that the organisation will likely adopt (Borgman et al. 2013: 4426). Innovation adoption can be divided into those that create incremental, synthetic or discontinuance change (Baker 2011, Hoti 2015). Incremental change is represented by the adoption of one version of an existing system to a newer version of the same system. Incremental change represents the least kind of risk and change for the adopting organisation. An example of synthetic change would be the delivery of existing paper-based context on a digital platform, requiring moderate change, combining existing ideas or technology with a novel one. Discontinuous changeis also regarded as radical change, for instance bar-codescanning that replaced price tags or the personal computer that replaced a mainframe computer. Discontinuous change could result in competence-enhancing or competence-destroying innovations and cause major shifts in industries (Baker 2011).

The organizational context is related to the characteristics and resources of the organisation, which includes employee structure, communication processes, size of the organisation and availability of resources (Borgman et al. 2013: 4427). Hoti (2015) is of the opinion that larger organisations can take greater risks associated with innovation adoption because they often have more resources and greater economies of scale. Greater adoption is achieved when informal linking agents such as champions are present to span cross-functional teams and employees. Organic and decentralised organisational structures are more conducive to innovation adoption, promoting lateral communication in addition to reporting lines. Innovation teams should be assembled that includes key employees from different sites and functions in the organisation to span functional silos (Baker 2011).

Structure of the industry, the presence of technology service providers and the regulatory environment makes up the environmental context (Borgman et al. 2013: 4428). Examples of the structure of the industry would for instance be intense competition or dominant firms that will influence other value chain partners. Rapidly growing industries are, according to Baker (2011) also more conducive to innovation adoption. On the contrary, organisations could also use a declining industry as incentives to expand into a new line of business and innovation adoption is then born from a desire to survive. Government regulations can either be beneficial to innovation, for example stringent safety requirements can deter innovation, while regulations such as pollution-control devices could mandate innovation adoption for an organisation (Baker 2011).

Hoti (2015) classified 87 scholarly articles that applied the TOE Framework in terms of innovation adoption and summarised the identified elements of the TOE Framework as follows: 
- Technological

i. Relative advantage: degree to which an innovation is perceived to be better than the idea it supersedes

ii. Compatibility: degree to which an innovation is perceived as consistent with existing values, past experiences and adopter needs

iii. Complexity: degree to which an innovation is perceived as relatively difficult to understand and use

- Organisational

i. Top management support: to the information system adoption initiative

ii. Organisational readiness: size, cost and technical resources, financial constraints and lack of professional expertise

iii. Information intensity and product characteristics: degree to which present information reflect level of information intensity of product or service

iv. Managerial time: time required to plan and implement new information system

- Environmental

i. Industry pressure: competition and high rivalry increases likelihood of innovation adoption to gain competitive advantage

ii. Government pressure/support: government strategies or initiatives that encourage innovation adoption

iii. Consumer readiness: customer readiness influences adoption process and information system usage

The paper henceforth introduces the research design and methods that were applied in understanding the technology, organisation and environment in which The Case organisation collects data and information relevant to sustainability reporting.

\section{Methodology}

The research project was approached from a pragmatic perspective. Pragmatism asserts that concepts only become relevant when they support action (Saunders et al. 2012:130). The ontology best applied to pragmatism is that of an external or multiple view, allowing the researcher to answer their research question (Saunders et al. 2012: 140). Pragmatic researchers view an idea's practical consequences as the importance of the meaning of an idea or research findings (Saunders et al. 2012: 130).

Qualitative research was deemed applicable in order to socially construct the nature of the reality at The Case. An inductive approach was used to gather 
data through purposive sampling of key employees, five from The Case and two from The Peer organisation. The participants for this study included managers of a number of business units who are directly involved in the reporting of sustainability data needed for strategic decision-making. Participants included the sustainability manager, operations manager and data capturer who report from a remote geographical location to head office. Interviews at the head office included the field and sustainability director as well as the regional financial manager. Two interviewswere conducted with the group sustainability coordinator and information technology specialist to determine the baseline for the MIS benchmarking at The Peer organisation.

The research strategy was based on evaluation research, using organizational documents as well as data collected from the interviews to establish the information flows and reporting processes into the various centralised systems from certain remote locations. The Peer organisation interviews were conducted to investigate the best practices for information flows and reporting into a MIS in order to recommend requirements and quick wins to streamline the reporting process of management information for The Case.Access to the organisations and permission to conduct the research were negotiated beforehand and the necessary consent forms declaring the principles of confidentiality and anonymity in terms of ethical research practices were completed and adhered to at all times.

\section{Findings}

The interviews from The Case organisation provided insight into the current information flows that takes place on a monthly basis between the head office and the 33 geographically dispersed lodges. This section reports firstly on the data flows, then highlights what is currently working well and points out the current challenges. The discussion is then followed by The Peer organisation's data flows after which recommendations are made for streamlining The Case organisation.

\section{Information Flows at the Case Organization}

All communication of the sustainability documents takes place using email attachments. Figure 1 provides a graphical representation of the typical document versions and information flows of The Case organisation. This flow diagram represents the data captured and exchanged from one lodge to the head office. When considering the complexity of the information flows, it is necessary to multiply this figure to represent 33 lodges. 
Vol. 5, No. 3 Mearns: Streamlining Management Information Systems Processes...

Figure 1. Sustainability Data, Document and Version Flows for the Case

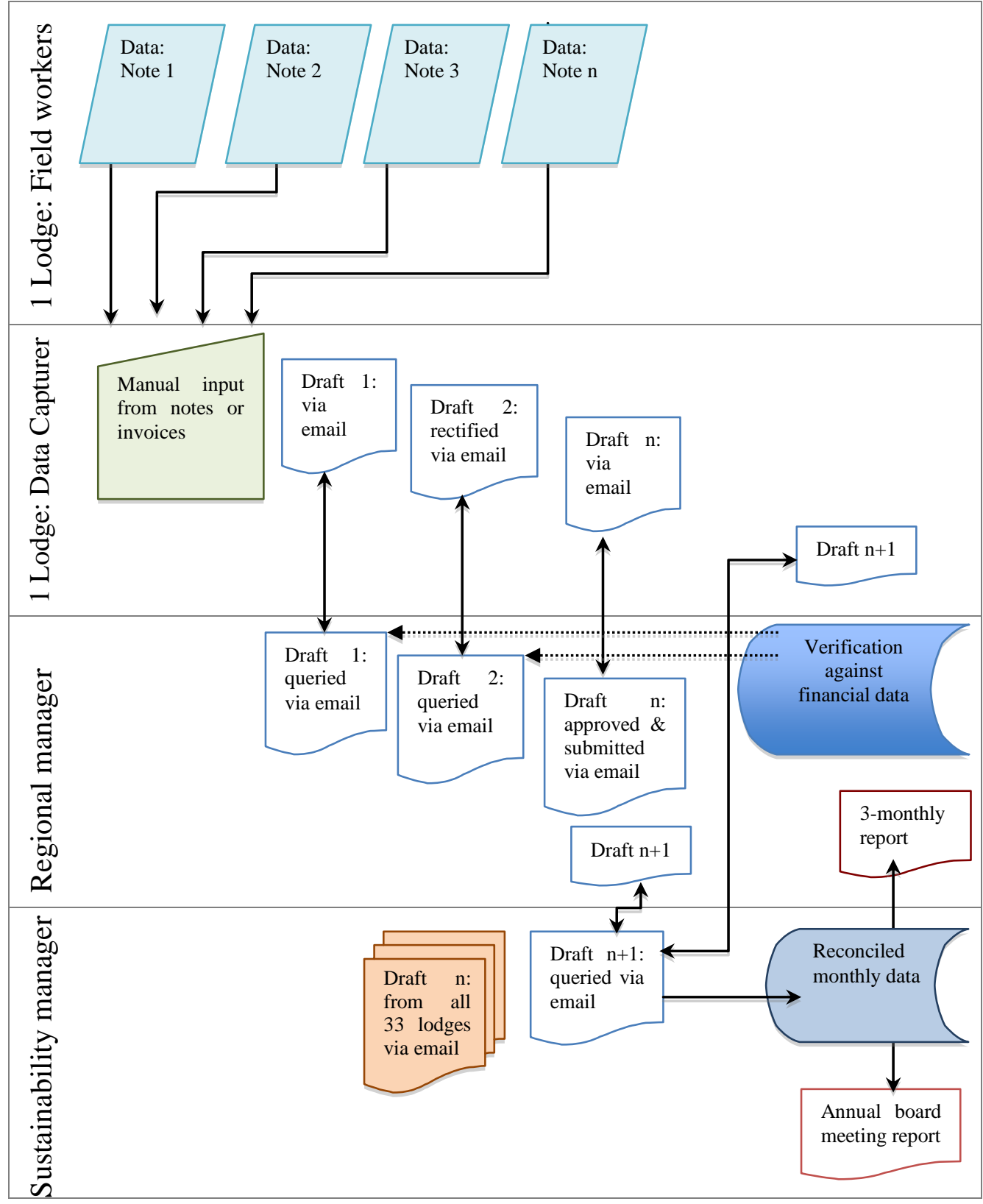

Source: Author.

On the second of each month, lodges are responsible to collect and capture data on various sustainability matters including water and electricity meter readings, fuel consumption per vehicle, diesel and petrol stocks and usage from tanks and bowsers, paraffin gas and charcoal usage and stocks. These readings are either supplied through invoices such as municipal accounts, day sheets or written down on notes as drivers fill vehicles or field workers are sent to read meters. Each lodge has a dedicated data capturer, although the employee also has many other roles and function to fulfill and is usually the lodge administrator, front office manager or lodge manager. The data is captured on MS Excel using terminals 
connected to the lodge or reserve server. Data capturing is done using units and currency although currency fluctuations and price variations on specifically fuel causes discrepancy in the data. Between the regional manager and the data capturer, the data is verified against financial data measuring actual to actual usage as well as actual to budget. The financial data is set up in terms of expenses, budgeted guest headcounts in terms of predicted occupancy and actual staff numbers present in the lodges. Numerous versions of the MS Excel file are sent via email between the 33 lodges and their four regional managers, until all queries are resolved. By the tenth of each month the 33 approved files are sent to the sustainability manager. If further queries are required, these are followed up via the regional manager or directly with the lodge data capturer and one monthly file, with all lodge sustainability data is collated. This file with the monthly data is reconciled and stored and a three-monthly report is sent to the regional manager and an annual report is generated for the board.

\section{Successes of the Current System at the Case Organization}

Immediately prior to the interviews, The Case organization changed the process of data collection from a cumbersome database run on MS Access to the MS Excel that is now in use. Data capturers reported that the MS Excel spreadsheets are user friendly, especially from the data input perspective. The system has not yet been used to its fullest extent, specifically in terms of the analytical functionalities. Fleet management and one region monitoring driver performance through the collected sustainability data are the exceptions to the system's analytical functionality use.

The interviewees reported a behaviour change and an increased awareness amongst staff since the sustainability data has been collected. The collected data has also put The Case organisation in a position to resolve challenges because there is data available as evidence and support. Because the information is collected and available, decisions can be made and anomalies can be explained.

The purpose for The Case organisation to report has also been achieved. Firstly the purpose was to create a data set in which intelligent decisions can be made. Furthermore, it was necessary to create awareness to consume less and as such, reduce the environmental impact. In the process ofreducing consumption, a cost reduction is a secondary advantage. It is also now possible to measure the highest cost sustainability aspects and reduce those as a priority. The Case organisation now strives to link guest occupancy with sustainability matters, meaning that if the lodge has 35\% occupancy then there should be a reduced environmental footprint when compared to a $95 \%$ occupancy rate. Occupancy rates are available but hasto be manually input from one system into the sustainability system. The Case organisation would also liketoget to a point where each vehicle or generator or water purification plant is monitored, to determine its efficiency and to make management decisions. 


\section{Challenges of the Current System at the Case Organisation}

The challenges with the current system at The Case organisation is discussed in terms of the TOE framework.

\section{$\underline{\text { Technological Challenges }}$}

As previously discussed in the literature review, technological aspects in the TOE framework refers to existing technologies in an organisation and the level of change needed to adopt new technologies. MS Excel is a system used on a regular basis by computer literate staff. The software is therefore somewhat familiar in The Case organisation and adoption of the software is not the matter that poses a challenge. The technological challenges lie in the frustration that staff reported with the duplication of having two systems, one on financial spreadsheets and one on sustainability spreadsheets. This is added to daily sheets and the withdrawal of data from one sheet to the other. When asked if this would be regarded as the priority improvement to make the interviewee's life easier the response was: "it is what it is, we just work harder".

The accuracy of the data is also deemed a concern. Each time manual data input is done, the accuracy of the data is at risk. Although perhaps little can be done to automate meter readings each time data is manually input from one system to another, there is a margin of data capture error that has to be considered. This is evident in the errors that have been reported by the interviewees in the opening and closing stock records. This has a negative impact on the analysis of the data as analysis cannot be done on erroneous data. Although Figure 1 shows the presence of many checks and balances in validating, querying and correcting data to ensure that reconciled data is stored, the manner in which the data sharing takes place at The Case organisation adds to the challenges.

Datasets are shared via email attachments and one interviewee called this "The Waiting Game". The data sits in someone's email inbox and this becomes a process challenge when the staff member is on leave. The matter of leave is discussed under the organisational challenges that follow. Wi-Fi connectivity and signal strength is a problem in many of the geographically remote locations which makes fully online systems an additional challenge. Although The Case organisation has made investments in terms of Wi-Fi infrastructure like antennae in some of these remote locations the signal strength remains an aspect beyond their control. The signal frustrations further challenges connectivity to shared drives and remote servers in some areas. Consultation with specialists is recommended in this regard. However, where user behaviour and process adjustments can be made to solve the problems, these should be carried out and managed as a priority as indicated in the organisational challenges section.

\section{Organizational Challenges}

Organizational aspects in the TOE Framework include employee capability, organizational structure, communication and processes and availability of 
resources. Top management support, organisational readiness and the intensity of the information as well as the managerial time to plan and implement technological innovations all impact on the challenges faced.

User behaviour change to safeguard organisational data can be managed through identifying the necessary capacity and mandating champions to push the boundaries towards improved processes and practices. One interviewee reported that besides reconciled data sets sitting in someone's email inbox, "they are saved somewhere on the server, everyone saves it somewhere else". Although this reflects negatively on user behaviour, it could very possibly be as a result of information overload and email fatigue those results in such behaviours. It could also be attributed to no standard way of saving documents and the absence of an organisational taxonomy, which essentially speaks to no or little information management governance.

Lodge employees specifically deal with a multitude of issues on a daily basis. Administrative tasks seem to sometimes pale in comparison to the immediate attention that guests and staff matters require on a daily basis. Furthermore, because of the remote locations of lodges, they are required to operate on varied leave cycles. These leave cycles vary between six-weeks-on-two-weeks-off, to three-months-on-one-month-off, to three-weeks-on-one-week-off. The cycles depend on whether the staff is local and live close to the lodge or whether they are expatriates and live far from the lodge. These varied leave cycles pose a process challenge in terms of querying and verifying the data as well as proper hand-over.

Another aspect that complicates reporting is where vehicles are occasionally borrowed between lodges. Whereas, the financial reporting is attached to which lodge owns the vehicle, the sustainability reporting is aligned with which lodge uses the vehicle. Fluctuations in the use of actual costs versus average costs also impacts on the accuracy of the data. This is mostly related to the purchasing of bulk fuel at a certain price and costs are then compared to fuel use at a different price after price fluctuations were announced. While the fluctuations in fuel price is a challenge associated with governmental pressure, The Case organisation dealt with this issue by working out a weighted average for fuel costing specifically.

\section{Environmental Challenges}

To reiterate the literature review, environmental aspects from the TOE Framework concenrs industry and governmental pressure as well as customer readiness. The tourism industryis under increased pressure to manage responsibly as was seen in the introduction to this paper in terms of the indicators, guidelines and standards to which tourism organisations should manage. Tourism organisations often tell stories to market the manner in which they are managing responsibly. One of the challenges that a number of interviewees pointed out were that The Case organisation is not yet telling the stories of their sustainability management successes. In order to tell these stories, the organisation needs to decide on a framework to measure and evaluate in order to know how effective 
the implemented projects are. Best practice stories need to be collected and told. A platform is needed where the lessons learnt can be communicated, being transparent about the mistakes, solutions and improvements. Not only will these lessons learnt reflect how sustainability has influenced behaviour, but will also celebrate success and inform the future.

The competition in the tourism industry necessitated the need to investigate The Peer organisation in order to provide perspective and a comparison for The Case organisation in terms of sustainability reporting.

\section{Information Flows at the Peer Organization}

The Peer organization operates in a similar region to The Case organisation and reports on 48 lodges compared to the 33 lodges of The Case. Similar geographic remote locations result in connectivity issues that are present in both organisations operating in southern and eastern Africa as well as some island locations. The main difference between The Peer and The Case organisations is that The Peer is a dual listed company trading on more than one country's stock exchange and is thus required to do integrated reporting to comply. Furthermore, The Peer organisation has approximately double the staff complement that The Case organisation has.

The information flows at The Peer organisation is depicted in Figure 2. A custom built online dashboard was developed for The Peer. Each lodge sees only what is relevant to their operation on the dashboard. The only aspects reported on are: measured generator hours, diesel in litres for generators and vehicles, propane gas in kilograms, petrol and paraffin in litres, wood in kilograms, grid and hydro-electricity in kilowatt hours, water in cubic metres and bottled water in litres. A total of eleven readings are input on the tenth of the month. This information is relevant to the environmental management systems (EMS) and is used to mitigate negative impacts on the environment. All other sustainability information such as community, biodiversity and financial aspects, are recorded seperately. The aim of the EMS recording is reported by The Peer to focus on EMS and not "blur the line with operations". These metrics result in the reporting of carbon footprints etc. although the report includes a lot more information and details apart from what is mentioned on the dashboard above.

The online dashboard then automatically generates an MS Excel spreadsheet which is checked by each of the three regional environmental managers. Any discrepancies are queried between the regional environmental manager and the data capturer at the lodge. A consolidated report is then sent to the group sustainability coordinator at a main office, ready for monitoring to ensure that strategies are effective and that the lodges are making progress towards these strategies. Data is used to rapidly identify areas of weakness or problems at camp or country level, and the data is stored until it is required for the annual integrated reporting. The sustainability data is used to report on the environmental footprint per guest bed night. 
Figure 2. Sustainability Data, Document and Version Flows for the Peer

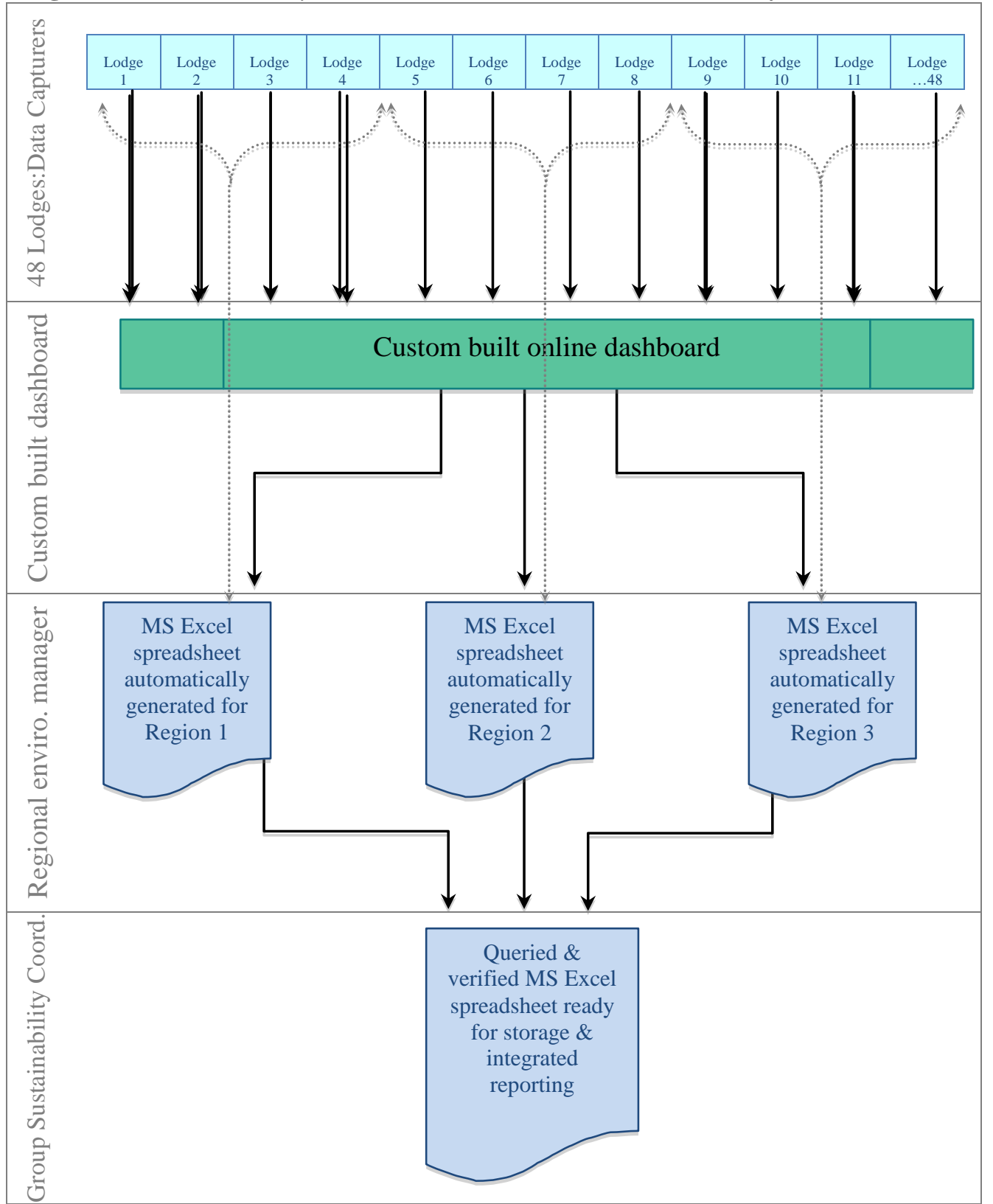

Source: Author.

Feedbacks to lodge managers are only done when there is a problem that has been identified in the data or when a specific lodge is excelling in a certain aspect. This is done to avoid information overflow, but at the same time to address problems where they exist and to celebrate successes or learn from a success. The data is not applied to do fleet management or to determine if a vehicle needs to be replaced, as this remains a regional operations department decision. The data is used to continually review and ensure that strategies are effective and if and where changes are needed. Through this data, decisions around the mitigation of negative environmental impacts or problems such as leaks or 
under-performing solar panels are possible. The data can therefore form the baseline to set up indicators or to monitor the four-year sustainability strategy. For instance, if the sustainability strategy indicated that $\mathrm{CO} 2$ emissions per bed nightneeds to be reduced to a certain percentage at a certain point in time, then the sustainability data is used to measure whether this specific reduction has taken place or to what extent it has been reached.

\section{Successes at the Peer Organization}

The Peer organisation has a very clear purpose for collecting sustainability data. They have developed a Group Environmental Minimum Standard to which all their lodges and operations should adhere. This standard is in place for everyone to work towards. At the time of the interviews they were $80 \%$ compliant with this standard. The regional environmental managers are handson which alleviates the pressure on camp managers. However, environmental reporting is part of the key performance indicators of regional operations departments and there is as a result, buy-in into the extra work expected from these relevant departments.

Since 2011 the data has been recorded and inroads are constantly made into data accuracy. Data accuracy is measured in comparison to bed-night reports. Indicators were initially extrapolated per guest night. The data is reported to correlate with these extrapolations more and more. The Group Environmental Minimum Standard requires short and long term strategies on sustainability matters and lodges are audited twice a year, once in the dry season and once in the wet season. The EMS data, as indicated on the dashboard used by The Peer, is a component of conservation alongside with biodiversity conservation. Besides conservation the other aspects reported in the sustainability matters are community, culture and commerce. This research only considered the reporting of EMS data.

\section{Challenges at the Peer Organization}

Some challenges were reported in terms of human error that creeps into the system which was ascribed to the number of links. However, these are minimal when considering Figure 2. Another challenge is the development of the custom-built dashboard. The developer has since left The Peer organisation and lack of knowledge retention has resulted that the new developer has little knowledge of how the system was built. At the time of the interviews the developer expressed concern with regards to changing anything on the system due to the current user satisfaction with the system. However, maintenance on the system is needed at times and the developer has to familiarise himself with the system.

Increased reporting accuracy is a continual organisation aspirational goal. When asked whether there is a requirement to measure staff usage separate from guest usage, the participant clearly stated that The Peer organisation does not see a need to separate guest and staff usage. The philosophy is that the staff 
is there as a result of the guest so the entire environmental footprint is ascribed to the presence of the guest. However, international benchmarks are conducted against bed nights splitting guest and staff impacts when measured against indicators (Baker and Mearns 2017:10, Styles et al. 2013: 615, Mearns 2010: 275). As a consequence The Peer and The Case organisations will not be in a position to benchmark internationally unless they are in a position to separate staff and guest impacts.

\section{Discussion}

When comparing Figure 1 and Figure 2 it is clear that there is a big difference in the processes of The Case and The Peer organisations. While The Peer's processes are more streamlined the major difference is due to the purpose for collecting the sustainability data. In The Case organisation the sustainability data is used to manage assets that have an environmental impact. In contrast, The Peer is monitoring sustainability data performance in terms of compliance with the sustainability strategy based on the Group Environmental Minimum Standard. The management of assets with an environmental impact at The Peer remains the responsibility of the lodge manager.

The first recommendation to The Case is therefore to use the historical data that has been collected to develop indicators and benchmarks to measure against. These indicators and benchmarks can be used to develop an organisational standard, similar to The Peer's Group Environmental Minimum Standard as the guiding document for sustainability strategies. At the time of the data collection The Case did not have sustainability performance tasks. In a 2017 publication of The Case, a vision for 2020 in terms of sustainability indicators was communicated. This is a step in the right direction and needs to be taken further.

In terms of the sustainability data collection process, innovation is required. Based on the observed organisational culture at The Case, staff is exceptionally loyal and hard working, willing to work harder when required. As stated in the literature review of this paper, innovation adoption could require incremental, synthetic or discontinuance change (Baker 2011, Hoti 2015). Incremental change is recommended with regard to The Case organisation due to the fact that the most recent change with the sustainability data collection was implemented within the past two years and the success achieved with the MS Excel data input deserves more momentum before large-scale changes are made. A shortterm quick-win change is therefore recommended to alleviate the number of document versions and email inbox congestions that was reported (Figure 1). The existing MS Excel document currently used for data collection should be placed on an online workflow and document management platform. A number of open source document management software (DMS) options exist (Maurya 2018) that needs to be investigated by The Case. The projected change in the information flow using one of these systems is depicted in Figure 3. 
Figure 3. Projected Change in Information Flow Using a Document Management System

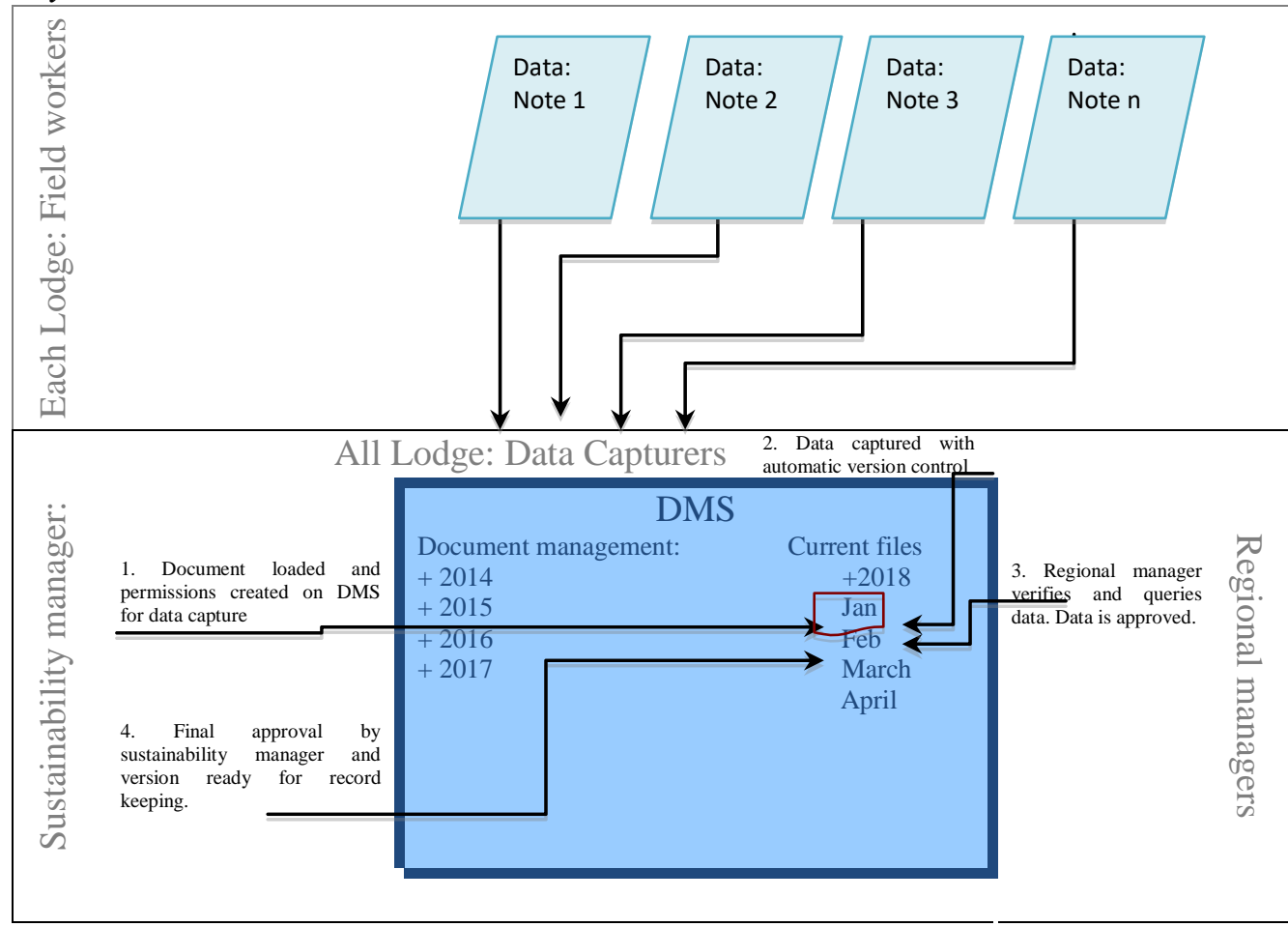

Source: Author.

The document can be loaded by admin. The lodge data capturer would be first in the workflow and checks out the document to input the data fields. The data capturer then checks the document back ready for the regional manager to check out the document for verification and validation purposes. The queries can be noted on the document and the versions are controlled within the DMS on the same document. When the document is approved by the regional manager the sustainability manager checks out the document and if no further queries are necessary the document is approved as final version and remains part of the DMS for record keeping purposes. With most DMSs files can be indexed as a result of the metadata created. Through the development of a taxonomy, the relevant documents are searchable which reduces the risk to projects and processes (Barquin 2006, Pincher 2010).

This suggestion is regarded as a quick-win for the organisation. In the longer term an integrated systems need to be investigated that can reduce the duplicate effort which the interview participants mentioned. With any of these systems, data connectivity needs to be evaluated and the least data intensive system may need to be chosen.

The process of sustainability data collection and sharing includes both tacit and explicit knowledge. The explicit knowledge is that which is codified and captured in the documents from which intelligent decisions will be made. The tacit knowledge lies within the process of collecting and managing the collection of the data. In the absence of key staff members, the process will grind to a halt. It is therefore imperative to develop a standard operating procedure 
to document the data collection process and practices so that better hand-over to relief staff or new staff can take place. This standard operating procedure will play a dual role in developing competency where necessary.

The loss of tacit knowledge was also mentioned by interview participants in the fact that the stories on sustainability issues are not being told. A platform is needed for the collection and dissemination of the stories and lessons learnt. Ihrig and MacMillan (2015: 87) points out those companies invest tens of millions of dollars to develop knowledge but pays hardly any attention whether the knowledge contributes to future competitive advantage. That is mostly due to the fact that many organisations have never mapped their mission-critical knowledge assets and are therefore not aware of what they know. "Knowledge moves individuals along the spectrum of uncertainty towards certainty; making risk a 'learnable' rather than an entirely random event" (Apgar 2006). Tacitness and complexity are associated with knowledge sources. Tacitness refers to the risk associated with managing the knowledge in people's heads, while complexity is determined by the amount of new knowledge necessary to manage the risk (Massingham 2010). The risk to knowledge that remains stuck in people's heads and is highly complex carries intolerable risk factors. In The Case organisation, the tacit knowledge can be managed and the nature of the knowledge in the sustainability data collection is not that complex. There should therefore not be a high risk in knowledge loss, yet in the absence of managing the tacit knowledge the risk remains unacceptable.

\section{Conclusions}

The Case organisation is a people-centred organisation with dedicated staff that offers extraordinary experiences for their guests. At their core, the organisation has a respect and care for the wildlife and communities that they share their environment with. Their dedication and commitment to be a responsible custodian is evident in the manner in which they manage and interact with their resources. The organisational culture reflects self-sacrifice and loyalty to their product. Although this is a noble trait it is also a potentially destructive trait when employees work harder and harder. This paper touched on a few suggestions to present a smarter way of working. The risk to the organisation to neglect proper document management is to some extent immeasurable.

The paper highlighted the successes and challenges that the current system and process presents. The overview of the process flow offers an opportunity to reflect on the complexity of what is achieved on a monthly basis. The Peer organisation's information flow allowed a view into a lean process. Although the way in which the sustainability data is used in terms of management in the two organisations have slight differences.

The streamlining of this process is intended to alleviate unnecessary administrative tasks wasted in document version management, waiting for responses from overflowing inboxes and lost data due to misfiling. Hopefully the time saved can then be dedicated to that which matters most: to make a difference 
in the lives of those people that cross our paths, so that the people around the wildlife parks will maintain an interest in them and the parks will survive.

\section{References}

Apgar D (2006) Risk intelligence: learning to manage what we don't know. Boston: Harvard University Press.

Baker J (2011) The technology-organization-environment framework. In YK Dwivedi (ed), Information systems theory: explaining and predicting our digital society 1 , Integrated series in information systems 28. doi 10.1007/978-1-4419-6108-2_12.

Baker MSP, Mearns K (2017) Applying sustainable tourism indicators to measure the sustainability performance of two tourism lodges in the Namib Desert. African Journal of Hospitality, Tourism and Leisure 6(2): 1-22.

Barquin R (2006) Managing risk with knowledge management. Retrieved from https://bit.ly/2MJBzUW.

Borgman HP, Bahli B, Heier H, Schewski F (2013) Cloudrise: exploring cloud computing adoption and governance with the TOE framework. 46th Hawaii International Conference on System Sciences (Wailea, [Maui], Hawaii, USA, 7 - 10 January 2013). doi 10.1109/HICSS.2013.132.

FTSE Russel (2016) ESG ratings and data model: integrating ESG into investments. Retrieved from https://bit.ly/2tjKnbe.

Hoti E (2015) The technological, organisational and environmental framework of IS innovation adaption in small and medium enterprises: evidence from research over the last 10 years. International journal of business management 3(4). doi 10.20472/BM.2015.3.4.001.

Hunter C, Mearns KF (2014) Assessing the sustainability reporting of selected tourism companies listed on the Johannesburg Stock Exchange (JSE). Africa journal of hospitality, tourism and leisure 4(1): 1-18.

Ihrig M, MacMilan I (2015) Managing your mission-critical knowledge: how to identify, map and leverage some of you company's most strategic assets. Harvard Business Review (Jan-Feb 2015): 81-87.

JSE (2015) Announcement: JSE Announces ESG Partnership with FTSE Russell. Retrieved from https://bit.ly/2tcfQwZ.

Laudon KC, Laudon JP (2018) Management Information Systems: Managing the Digital Firm, 15th ed. Edinburgh: Pearson.

Massingham P (2010) Knowledge risk management: a framework. Journal of knowledge management 14(3): 464-485.

Maurya R (2018) Top 12 free open source document management system software. Retrieved from https://bit.ly/2rRUNBD.

Mearns KF (2010) The sustainability of community-based ecotourism in southern Africa. Unpublished PhD Thesis, University of Portsmouth, UK, 365pp.

Pincher M (2010) A guide to developing taxonomies for effective data management. Computerweekly. Retrieved from https://bit.ly/2JQymoN. [Accessed March 2010]

SANS (2016) Responsible tourism - requirements. Retrieved from https://bit.ly/2tki9xe.

Saunders M, Lewis P, Thornhill A (2012) Research Methods for Business Students. Harlow, Pearson Education Ltd.

Shiau W, Chen S, Tsai Y ( 2015) Management information systems issues: co-citation analysis of journal articles. International journal of electronic commerce studies 6(1): 145-162. doi 10.7903.ijecs.1393. 
Styles D, Schönberger H, Martos JLG (2013) Best Environmental Management Practice in the Tourism Sector: Learning from frontrunners. European Commission, Joint Research Centre, Institute for Prospective Technological Studies.Retrieved from https://bit.ly/2M9umgb.

The Global Reporting Initiative (2018) The Global Reporting Initiative. Retrieved from https://bit.ly/1UL5UAS.

Zwass V (1984) Management information systems: beyond the current paradigm. Journal of management information systems 1(1): 3-1. 
